\title{
AN ALMOST-SURE RENEWAL THEOREM FOR BRANCHING RANDOM WALKS ON THE LINE
}

\author{
MATTHIAS MEINERS, ${ }^{*}$ Uppsala University
}

\begin{abstract}
In the present paper an almost-sure renewal theorem for branching random walks (BRWs) on the real line is formulated and established. The theorem constitutes a generalization of Nerman's theorem on the almost-sure convergence of Malthus normed supercritical Crump-Mode-Jagers branching processes counted with general characteristic and Gatouras' almost-sure renewal theorem for BRWs on a lattice.
\end{abstract}

Keywords: Branching random walk; martingale; renewal theorem

2010 Mathematics Subject Classification: Primary 60J80

Secondary $60 \mathrm{~K} 05$

\section{Introduction}

Almost-sure limit theorems for general (Crump-Mode-Jagers) branching processes, which can be viewed as branching random walks (BRWs) with positive increments, have proven useful in the theory of branching processes. For instance, Nerman's almost-sure renewal theorem [13, Theorem 5.4] has been successfully applied in the study of the functional equation in the BRW; see, e.g. [3, Proposition 9.2] and [4, Theorem 8.6]. For another example of a fruitful application of almost-sure renewal theorems for BRWs is the area of random fractals, see [7]. In this paper we address the problem of extending the almost-sure renewal theorems for BRWs on the positive half-line to BRWs on the whole real line.

The rest of this paper is organized as follows. In Section 2 we give an introduction to the BRW and the notation used throughout the paper. Our main results are stated in Section 3. Some results from classical renewal theory are collected in Section 4. In Sections 5 and 6 known results on connections between BRWs and their associated random walks, and the corresponding ladder line and ladder height processes, respectively, are summarized. Section 7 contains the proof of our main result, the almost-sure renewal theorem for BRWs.

\section{Notation and preliminaries}

We begin this section with an introduction to the BRW on the real line. Consider an individual, the ancestor, which we identify with the empty tuple $\varnothing$, located at the origin of the real line at time $n=0$. At time $n=1$ the ancestor produces a random number, $N$, of offspring which are placed at real points according to a random point process $\mathcal{Z}=\sum_{i=1}^{N} \delta_{X_{i}}$ on $\mathbb{R}$ (particularly, $N=\mathfrak{Z}(\mathbb{R})$ ). Here $\delta_{x}$ denotes the Dirac measure with a point at $x$. We enumerate the ancestor's children by $1,2, \ldots, N$ (note that we do not exclude the case in which $N=\infty$ with positive probability). The offspring of the ancestor form the first generation.

Received 23 February 2010; revision received 7 June 2010.

* Postal address: Department of Mathematics, Uppsala University, 75106 Uppsala, Sweden.

Email address: matthias.meiners@math.uu.se

Research supported by DFG grant Me 3625/1-1. 
The population further evolves following the subsequently explained rules. An individual $v=\left(v_{1}, \ldots, v_{n}\right) \in \mathbb{N}^{n}$ of the $n$th generation with position $S(v)$ on the real line produces at time $n+1$ a random number, $N(v)$, of offspring which are placed at random locations on $\mathbb{R}$ given by the positions of the random point process $\delta_{S(v)} * \mathcal{Z}(v)=\sum_{i=1}^{N(v)} \delta_{S(v)+X_{i}(v)}$, where $\mathcal{Z}(v)=\sum_{i=1}^{N(v)} \delta_{X_{i}(v)}$ denotes a copy of $\mathcal{Z}($ and $N(v)=\mathfrak{Z}(v)(\mathbb{R})$ ). The offspring of individual $v$ are enumerated by $\left(v_{1}, \ldots, v_{n}, 1\right), \ldots,\left(v_{1}, \ldots, v_{n}, N(v)\right)$, the positions of offspring individuals are denoted by $S\left(\left(v_{1}, \ldots, v_{n}, i\right)\right), i=1, \ldots, N(v)$. It remains to state that $(\mathcal{Z}(v))_{v \in \mathbb{V}}$ is assumed to be a family of independent and identically distributed (i.i.d.) point processes, where $\mathbb{V}$ is the set of all potential individuals of the process. The point process of the positions of the $n$th generation individuals will be denoted by $\mathcal{Z}_{n}$. The sequence of point processes $\left(\mathcal{Z}_{n}\right)_{n \geq 0}$ is then called a BRW.

The set of potential individuals of the $n$th generation is given by $\mathbb{N}^{n}, n \geq 0$, where $\mathbb{N}^{0}$ is defined to be the singleton of the empty tuple, i.e. $\mathbb{N}^{0}=\{\varnothing\}$. The set of all potential individuals of the process is given by $\mathbb{V}=\bigcup_{n \geq 0} \mathbb{N}^{n}$. In what follows, we stick to the usual Ulam-Harris notation, which means that we abbreviate individuals $v=\left(v_{1}, \ldots, v_{n}\right) \in \mathbb{V}$ by $v_{1} \cdots v_{n}$ so that $v w$ is an abbreviation for the individual $\left(v_{1}, \ldots, v_{n}, w_{1}, \ldots, w_{m}\right)$ when $w=\left(w_{1}, \ldots, w_{m}\right) \in \mathbb{N}^{m}$. We understand $|v|$ to be the generation in which $v$ resides. The ancestor of $v$ in the $k$ th generation, $\left(v_{1}, \ldots, v_{k}\right)$, will be denoted by $v \mid k$ for $k \leq n$. In particular, $v \mid 0=\varnothing$. We write $u \prec v$ to indicate that $u$ is a strict ancestor of $v$, which means that there exists some $k<n$ such that $u=v \mid k$. We write $u \preceq v$ if either $u \prec v$ or $u=v$, i.e. if $u$ is an ancestor of $v$. For subsets $V \subseteq \mathbb{V}$, we write $u \prec V$ if no $v \in V$ is an ancestor of $u$, i.e. if $v \npreceq u$ for all $v \in V$.

The basic probability space is defined to be the product space

$$
(\Omega, \mathcal{A}, \mathrm{P})=\prod_{v \in \mathbb{V}}\left(\Omega_{v}, \mathcal{A}_{v}, P_{v}\right),
$$

where $\left(\Omega_{v}, \mathcal{A}_{v}, P_{v}\right)$ are identical spaces. We suppose that $\left(\Omega_{v}, \mathcal{A}_{v}, P_{v}\right)$ carries the point process $\mathcal{Z}(v)$, but may also carry further random variables. Since $(\Omega, \mathcal{A}, \mathrm{P})$ is a product space, an element $\omega \in \Omega$ is of the form $\omega=\left(\omega_{v}\right)_{v \in \mathbb{V}}$. For each $u \in \mathbb{V}$, let $\sigma_{u}: \Omega \rightarrow \Omega$, $\omega=\left(\omega_{v}\right)_{v \in \mathbb{V}} \mapsto \sigma_{u} \omega:=\left(\omega_{u v}\right)_{v \in \mathbb{V}}$ be the shift operator. Whenever $\Psi$ is a function from $(\Omega, \mathcal{A})$ into another measurable space, we denote by $[\Psi]_{u}$ the function $\omega \mapsto \Psi\left(\sigma_{u} \omega\right)$.

We assume throughout the paper that the average number of children born to each individual is greater than 1, that is, $\mathrm{E} N>1$. In other words, we assume the supercriticality of the underlying branching process $\left(N_{n}\right)_{n \geq 0}$, where $N_{n}$ is defined to be the number of realized individuals of the $n$th generation, a formal definition of which is given next. Set $g_{0}:=\{\varnothing\}$ and, recursively,

$$
g_{n+1}:=\left\{v i \in \mathbb{N}^{n+1}: v \in g_{n}, 1 \leq i \leq N(v)\right\}, \quad n \in \mathbb{N}_{0},
$$

and, finally, $g:=\bigcup_{n \geq 0} g_{n}$. Then, $N_{n}:=\left|g_{n}\right|, n \geq 0$. Here $\left(N_{n}\right)_{n \geq 0}$ forms a Galton-Watson branching process if $\bar{N}$ is almost surely (a.s.) finite. The assumption that $\mathrm{E} N>1$ guarantees that $\mathrm{P}(S)>0$, where $S$ is defined to be the survival set of the process,

$$
S:=\left\{N_{n}>0 \text { for all } n \geq 0\right\} .
$$

By $\xi$ we denote the intensity measure of the point process $\mathbb{Z}$, i.e. $\xi(A):=\mathrm{E} \mathcal{Z}(A)$ for any Borel set $A \subseteq \mathbb{R}$. Furthermore, we define $m$ to be the Laplace transform of $\xi$, i.e., for $\theta \in \mathbb{R}$,

$$
m(\theta):=\int \mathrm{e}^{-\theta x} \xi(\mathrm{d} x)=\mathrm{E} \int \mathrm{e}^{-\theta x} \mathcal{Z}(\mathrm{d} x)=\mathrm{E} \sum_{i=1}^{N} \mathrm{e}^{-\theta X_{i}} .
$$


Note that, by nonnegativity, $m$ is well defined on $\mathbb{R}$ but may assume the value $+\infty$. We write $\mathfrak{D}(m)$ for the canonical domain of $m$ defined by $\mathfrak{D}(m):=\{\theta \in \mathbb{R}: m(\theta)<\infty\}$. Since $m$ is a Laplace transform, $\mathfrak{D}(m)$ is a convex subset of $\mathbb{R}$, but may in general be empty or contain an infinite ray. In what follows, we make two substantial assumptions concerning $m$.

(A1) There exists an $\alpha>0$ such that $m(\alpha)=1$.

Under (A1), the convexity of $m$ implies that the equation $m(\theta)=1$ has either one or two solutions. Since we assume that $\mathrm{E} N=m(0)>1$, any solution to the equation is positive. Henceforth, we assume that $\alpha$ is the minimal solution to the equation $m(\theta)=1$. Our second assumption is

(A2) $-m^{\prime}(\alpha) \in(0, \infty)$, where $-m^{\prime}(\theta):=\mathrm{E} \sum_{i=1}^{N} X_{i} \mathrm{e}^{-\theta X_{i}}=\int x \mathrm{e}^{-\theta x} \xi(\mathrm{d} x)$.

Particularly, validity of (A2) implies that $\sum_{i=1}^{N} X_{i} \mathrm{e}^{-\alpha X_{i}}$ is integrable. Note that $m$ is differentiable on $\operatorname{int}(\mathfrak{D}(m))$ and that $m^{\prime}(\theta)$ as defined above coincides with the derivative of $m$ on $\operatorname{int}(\mathfrak{D}(m))$. It is well known that assumption (A1) makes the sequence of (random) Laplace transforms of the random point measures $\left(Z_{n}\right)_{n \geq 0}$ evaluated at $\theta=\alpha$ a nonnegative martingale with respect to the filtration

$$
\mathcal{F}_{n}:=\sigma\left(\mathcal{A}_{v}:|v|<n\right), \quad n \geq 0 .
$$

(Note the slight abuse of notation in (2.1), where $\mathcal{F}_{n}$ is understood to be the $\sigma$-algebra generated by the projections $p_{v}: \Omega \rightarrow \Omega_{v}, \omega=\left(\omega_{u}\right)_{u \in \mathbb{V}} \mapsto \omega_{v},|v|<n$.) We denote this martingale by $\left(W_{n}^{(\alpha)}\right)_{n \geq 0}$, i.e.

$$
W_{n}^{(\alpha)}:=\int \mathrm{e}^{-\alpha x} \mathcal{Z}_{n}(\mathrm{~d} x)=\sum_{|v|=n} \mathrm{e}^{-\alpha S(v)}, \quad n \geq 0,
$$

where here and in the following the summation over $|v|=n$ means the summation over $v \in g_{n}$. Since being nonnegative, $\left(W_{n}^{(\alpha)}\right)_{n \geq 0}$ converges a.s. to a nonnegative limit which we denote by $W^{(\alpha)}$. An equivalent criterion (under (A1) and (A2)) for $W^{(\alpha)}$ not to be degenerate at 0 is next.

Theorem 2.1. (Biggins' theorem.) In the given situation, the following assertions are equivalent.

(a) $\left(W_{n}^{(\alpha)}\right)_{n \geq 0}$ is uniformly integrable.

(b) $W_{n}^{(\alpha)} \rightarrow W^{(\alpha)}$ in $\mathcal{L}^{1}$ as $n \rightarrow \infty$.

(c) $\mathrm{E} W^{(\alpha)}=1$.

(d) $\mathrm{P}\left(W^{(\alpha)}>0\right)>0$.

(e) $\left\{W^{(\alpha)}>0\right\}=S$ a.s.

(f) $\mathrm{E} W_{1}^{(\alpha)} \log ^{+} W_{1}^{(\alpha)}<\infty$.

Proof. See [12] for a conceptual proof.

\section{Almost-sure renewal theorems for BRWs on the line}

In addition to the BRW as defined above, we suppose the existence of a product-measurable, separable stochastic process $\phi: \mathbb{R} \times \Omega \rightarrow \mathbb{R} \cup\{\infty\}$. In the context of Crump-Mode-Jagers processes, $\phi$ can be interpreted as a general characteristic of the process; see [13]. As usual, we 
suppress the dependence of $\phi$ on $\omega$ in most of the formulae, i.e. we write $\phi(t)$ and think of it as the random variable $\omega \mapsto \phi(t, \omega)$. We then define (cf. [9, p. 167] and [13, Equation (1.11)])

$$
Z_{t}^{\phi}:=\sum_{v}[\phi]_{v}(t-S(v))
$$

where here and in what follows $\sum_{v}$ means the summation over $g$, the set of realized individuals.

For technical reasons, we have to distinguish two cases, the lattice and the nonlattice cases. We call $\mathcal{Z}$ lattice if, for some $r>0, \mathrm{P}(\mathcal{Z}(\mathbb{R} \backslash r \mathbb{Z})=0)=1$, and nonlattice if no such $r>0$ exists. In the lattice case, we refer to $\lambda>0$ as the lattice span if

$$
\lambda=\sup \{r>0: \mathrm{P}(\mathcal{Z}(\mathbb{R} \backslash r \mathbb{Z})=0)=1\} .
$$

Next, we introduce two assumptions, which we need to prove the renewal theorem for BRWs on the line. The first condition is a moment condition for the positive steps of the BRW while the second condition affects the stochastic process $\phi$ and the negative steps of the BRW.

Condition 3.1. There exists a nonincreasing and integrable function $g:[0, \infty) \rightarrow(0, \infty)$ such that

$$
\mathrm{E} \sum_{|v|=1} \frac{\mathrm{e}^{-\alpha S(v)}}{g(S(v))} \mathbf{1}_{\{S(v) \geq 0\}}<\infty .
$$

Condition 3.2. Let $\varepsilon>0$, and define the function $h$ by $h(0)=1$ and

$$
h(t):=\min \left\{1,|t|^{-1}\left(\log ^{+}|t|\right)^{-(1+\varepsilon)}\right\}, \quad t \neq 0 .
$$

Then

$$
M:=\sup _{t \in \mathbb{R}} \frac{\mathrm{e}^{-\alpha t}|\phi(t)|}{h(t)} \in \mathcal{L}^{1}
$$

and

$$
\mathrm{E} \sum_{|v|=1} \mathrm{e}^{-\alpha S(v)} \frac{|S(v)|}{h(S(v))} \mathbf{1}_{\{S(v)<0\}}<\infty .
$$

Remark 3.1. (a) In the lattice case with lattice span $\lambda>0$, it suffices to assume integrability of $M_{s}=\sup _{n \in \mathbb{Z}} \mathrm{e}^{-\alpha(s+n \lambda)}|\phi(s+n \lambda)| / h(s+n \lambda)$ for all $s \in[0, \lambda)$ instead of the integrability of $M$.

(b) The moment assumption on the negative steps is stronger than the assumption affecting the positive steps. We need this stronger assumption in the proof of our main result to uniformly control the negative excursions of the BRW.

(c) The explicit form of the function $h$ in Condition 3.2 is not important. In fact, in our proofs we use the facts that $h$ is symmetric (which is for convenience only), nonincreasing, regularly varying at $\infty$ and integrable with respect to the Lebesgue measure, and that $1 / h$ is convex on $[0, \infty)$.

Now we are ready to present our main result. We split it into two theorems, the first concerning the nonlattice case, the second concerning the lattice case.

Theorem 3.1. (Nonlattice case.) Suppose that $\mathcal{Z}$ is nonlattice, that Conditions 3.1 and 3.2 are satisfied, and that $\phi$ a.s. has càdlàg paths. Then, as $t \rightarrow \infty$,

$$
\mathrm{e}^{-\alpha t} Z_{t}^{\phi} \rightarrow \frac{W^{(\alpha)}}{-m^{\prime}(\alpha)} \int_{\mathbb{R}} \mathrm{e}^{-\alpha s} \mathrm{E} \phi(s) \mathrm{d} s \quad \text { a.s. }
$$


Remark 3.2. Theorem 3.1 is a generalization of Theorem 5.4 of [13], except for the fact that our Condition 3.1 and the choice of $h$ in Condition 3.2 are slightly more restrictive than Condition 5.1 and the choice of $h$ in Condition 5.2 of [13], respectively.

In turn, Theorem 5.4 of [13] implies that Theorem 3.1 holds if $\mathcal{Z}$ is a.s. concentrated on the positive half-line, and if $\phi$ is nonnegative and vanishes on the negative half-line.

Theorem 3.2. (Lattice case.) Suppose that $\mathcal{Z}$ is lattice with span $\lambda>0$, and that Conditions 3.1 and 3.2 hold. Then, for any $s \in[0, \lambda)$, as $n \rightarrow \infty$,

$$
\mathrm{e}^{-\alpha n \lambda} Z_{n \lambda+s}^{\phi} \rightarrow \frac{\lambda W^{(\alpha)}}{-m^{\prime}(\alpha)} \sum_{k \in \mathbb{Z}} \mathrm{e}^{-\alpha k \lambda} \mathrm{E} \phi(k \lambda+s) \text { a.s. }
$$

Remark 3.3. Similar to the situation in the nonlattice case, Theorem 3.2 is a generalization of Theorem 3.2 of [8] with the same exception mentioned in Remark 3.2: Condition 3.1 and the choice of $h$ in Condition 3.2 are slighty more restrictive than Condition 3.2 and the choice of $h$ in Condition 3.1 of [8], respectively.

Again, Theorem 3.2 of [8] implies that Theorem 3.2 holds if $\mathcal{Z}$ is a.s. concentrated on the positive half-line, and if $\phi$ is nonnegative and vanishes on the negative half-line.

As a corollary from Theorems 3.1 and 3.2, we formulate a Blackwell-type result which provides information about the asymptotic number of individuals in the BRW residing in an interval of the form $[t, t+h]$ as $t \rightarrow \infty$ or in a singleton $\{n \lambda\}$ as $n \rightarrow \infty$.

Corollary 3.1. (Blackwell-type renewal theorem for BRWs.) Assume that assumptions (A1) and (A2) are satisfied and that, for some $\varepsilon>0$,

$$
\mathrm{E} \sum_{|v|=1} \mathrm{e}^{-\alpha S(v)}\left(1 \vee S(v)^{-}\right)|S(v)| \log ^{1+\varepsilon}(|S(v)|)<\infty .
$$

(a) Suppose that $\mathbb{Z}$ is nonlattice. Then, for any $h>0$, as $t \rightarrow \infty$,

$$
\#\{v \in g: S(v) \in[t, t+h]\} \sim \frac{\mathrm{e}^{\alpha h}-1}{\alpha} \frac{W^{(\alpha)}}{-m^{\prime}(\alpha)} \mathrm{e}^{\alpha t} \quad \text { a.s. }
$$

(b) Suppose that $\mathcal{Z}$ is lattice with lattice span $\lambda>0$. Then, as $n \rightarrow \infty$,

$$
\#\{v \in g: S(v)=n \lambda\} \sim \frac{\lambda W^{(\alpha)}}{-m^{\prime}(\alpha)} \mathrm{e}^{\alpha n \lambda} \quad \text { a.s. }
$$

Proof. Equations (3.8) and (3.9) immediately follow from (3.5) and (3.6) with $\phi(t):=$ $\mathbf{1}_{[-h, 0]}(t)$ or $\phi(t)=\mathbf{1}_{\{0\}}(t)$, respectively.

\section{An auxiliary result from renewal theory}

In this section we denote by $\left(S_{n}\right)_{n \geq 0}$ an arbitrary random walk $\left(S_{n}\right)_{n \geq 0}$ with positive drift $\mu$ and first strictly ascending ladder index $\sigma^{>}$. We denote by $V^{>}$the pre- $\sigma^{>}$occupation measure of $\left(S_{n}\right)_{n \geq 0}$, i.e. the measure

$$
V^{>}=\mathrm{E} \sum_{k=0}^{\sigma^{>}-1} \delta_{S_{k}}
$$

Since $\left(S_{n}\right)_{n \geq 0}$ is positive divergent, we have $\mathrm{E} \sigma^{>}<\infty$. Particularly, $V^{>}$is a finite measure. It is known in renewal theory that in the situation described above the normalized pre- $\sigma^{>}$ 
occupation measure $\left(\mathrm{E} \sigma^{>}\right)^{-1} V^{>}$equals the distribution of $\min _{n \geq 0} S_{n}$, the global minimum of the random walk, i.e.

$$
\left(\mathrm{E} \sigma^{>}\right)^{-1} V^{>}(\cdot)=\mathrm{P}\left(\min _{n \geq 0} S_{n} \in \cdot\right) .
$$

In the proofs of Theorems 3.1 and 3.2, we need insight about the finiteness of integrals over certain regularly varying functions with respect to the pre- $\sigma^{>}$occupation measure of a special random walk, the so-called associated random walk of the BRW. The investigation of these integrals will be arranged in Proposition 4.1 via connection (4.2).

Proposition 4.1. Let $f:[0, \infty) \rightarrow[0, \infty)$ denote a nondecreasing convex function which is regularly varying at $\infty$ of order $p \geq 1$. Furthermore, denote by $\sigma^{<}$the first strictly descending ladder index of the random walk $\left(S_{n}\right)_{n \geq 0}$, i.e. $\sigma^{<}=\inf \left\{n \geq 1: S_{n}<0\right\}$. Then the following assertions are equivalent.

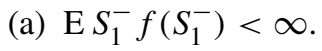

(b) $\operatorname{E~} f\left(\mid S_{\sigma<\mid}\right) \mathbf{1}_{\{\sigma<<\infty\}}<\infty$.

(c) $\mathrm{E} f\left(\left|\min _{n \geq 0} S_{n}\right|\right)<\infty$.

(d) $\int f(|s|) V^{>}(\mathrm{d} s)<\infty$.

From classical renewal theory it follows that Proposition 4.1(a)-(c) are equivalent for $f(t)=$ $t^{p}, p>1$; see, for instance, [11]. Due to connection (4.2), $\mathrm{E}\left(S_{1}^{-}\right)^{p+1}<\infty$ is also necessary and sufficient for the function $t \mapsto|t|^{p}$ to be $V^{>}$integrable. The transfer to functions $f$ as above is a generalization of the proof of Theorem 1 of [11]; see also the proof of [1, Satz 4.1.7].

Proof of Proposition 4.1. The equivalence between assertions (c) and (d) directly follows from (4.2). Therefore, it remains to prove that assertions (a), (b), and (c) are equivalent.

To this end, note that it constitutes no loss of generality to assume that $f$ is continuous on $[0, \infty)$. Then, since being continuous and convex, $f$ is absolutely continuous on $[0, \infty)$ and, thus, almost everywhere differentiable with respect to the Lebesgue measure. We denote by $f^{\prime}$ the right derivative of $f$, which is defined everywhere. Then, by the fundamental theorem of calculus for the Lebesgue integral, $f(x)-f(0)=\int_{0}^{x} f^{\prime}(t) \mathrm{d} t, x \geq 0$. To make this formula even simpler, we assume that $f(0)=0$, which can easily be arranged by replacing $f$ by $x \mapsto f(x)-f(0)$ without affecting the finiteness of the expectations in (a), (b), and (c). Then

$$
f(x)=\int_{0}^{x} f^{\prime}(t) \mathrm{d} t, \quad x \geq 0 .
$$

Next, we define $F(x):=\int_{0}^{x} f(t) \mathrm{d} t$ and note that $F(x) \sim x f(x) /(p+1)$ as $x \rightarrow \infty$ by the direct half of Karamata's theorem; see [6, Proposition 1.5.8]. Furthermore, by [11, Lemma 2], there exist positive constants $c, C>0$ such that

$$
\begin{aligned}
c \int_{t}^{\infty} \mathrm{P}\left(S_{1}^{-}>x\right) \mathrm{d} x & \leq c \sum_{j=0}^{\infty} \mathrm{P}\left(S_{1}^{-}>t+j\right) \\
& \leq \mathrm{P}\left(\left|S_{\sigma}<\right|>t, \sigma^{<}<\infty\right) \\
& \leq C \sum_{j=0}^{\infty} \mathrm{P}\left(S_{1}^{-}>t+j\right) \leq C \int_{t-1}^{\infty} \mathrm{P}\left(S_{1}^{-}>x\right) \mathrm{d} x
\end{aligned}
$$

for all $t \geq 0$. 
Now assume that (a) holds. Then, by (4.5), Fubini's theorem, and (4.3),

$$
\begin{aligned}
\operatorname{E} f\left(\mid S_{\sigma<\mid}\right) \mathbf{1}_{\left\{\sigma^{<}<\infty\right\}} & =\int_{0}^{\infty} f^{\prime}(t) \mathrm{P}\left(\left|S_{\sigma<}\right|>t, \sigma^{<}<\infty\right) \mathrm{d} t \\
& \leq C \int_{0}^{\infty} f^{\prime}(t) \int_{t-1}^{\infty} \mathrm{P}\left(S_{1}^{-}>x\right) \mathrm{d} x \mathrm{~d} t \\
& =C \int_{-1}^{\infty} f(x+1) \mathrm{P}\left(S_{1}^{-}>x\right) \mathrm{d} x \\
& =C \operatorname{EF}\left(S_{1}^{-}+1\right) \\
& <\infty,
\end{aligned}
$$

due to the fact that $F(x) \sim x f(x) /(p+1)$ as $x \rightarrow \infty$. We have thus shown that (a) implies (b). Conversely, if (b) holds, using the same calculations as above with (4.5) replaced by (4.4) and with ' $\leq$ ' replaced by ' $\geq$ ', we find that (b) implies (a) too.

Assume that (b) holds, and let $\left(\left(\tau_{k}, W_{k}\right)\right)_{k \geq 1}$ denote an i.i.d. sequence of random variables with $\left(\tau_{1}, W_{1}\right)$ distributed like $\left(\sigma^{<}, S_{\sigma}<\right)$ given $\sigma^{<}<\infty$. Then, due to [11, Lemma 1], with $T_{\min }:=\inf \left\{k \geq 0: S_{k}=\min _{n \geq 0} S_{n}\right\}$, we have

$$
\left(T_{\min }, \min _{n \geq 0} S_{n}\right) \stackrel{\mathrm{D}}{=}\left(\sum_{k=1}^{L} \tau_{k}, \sum_{k=1}^{L} W_{k}\right)
$$

where $L$ is independent of $\left(\left(\tau_{k}, W_{k}\right)\right)_{k \geq 1}$ with $\mathrm{P}(L=l)=\gamma(1-\gamma)^{l}, l \geq 0, \gamma=\mathrm{P}\left(\sigma^{<}=\infty\right)$, and ' $=$, denotes equality in distribution. Here, $\gamma>0$ since $\mu>0$. From the distributional identity (4.6), we infer by an application of Jensen's inequality that

$$
\begin{aligned}
\operatorname{E} f\left(\left|\min _{n \geq 0} S_{n}\right|\right) & =\sum_{l=1}^{\infty} \gamma(1-\gamma)^{l} \text { E } f\left(\sum_{k=1}^{l}\left|W_{k}\right|\right) \\
& \leq \sum_{l=1}^{\infty} \gamma(1-\gamma)^{l} \operatorname{E} f\left(l\left|W_{1}\right|\right) .
\end{aligned}
$$

By Potter's theorem [6, Theorem 1.5.6(iii)], there exists some $c>0$ such that $f(y) / f(x) \leq$ $2(y / x)^{p+1}$ for any $x, y \geq c$. Thus, for any $l \geq 1$,

$$
f\left(l\left|W_{1}\right|\right)=\frac{f\left(l\left|W_{1}\right|\right)}{f\left(\left|W_{1}\right|\right)} f\left(\left|W_{1}\right|\right) \leq 2 l^{p+1} f\left(\left|W_{1}\right|\right)+f(l c) .
$$

Thus, the expectations in (4.7) grow at most polynomially fast in $l$. Hence, due to assumption (b), which implies the finiteness of $\mathrm{E} f\left(\left|W_{1}\right|\right)$, the series in (4.7) converges. On the other hand, that (c) implies (b) immediately follows from the inequality $\left|S_{\sigma<}\right| \leq\left|\min _{n \geq 0} S_{n}\right|$ on $\left\{\sigma^{<}<\infty\right\}$.

\section{The link to renewal theory}

In the theory of BRWs it is folklore that-provided there exists a Malthusian parameterthere is a link to renewal theory via the associated random walk $\left(S_{n}\right)_{n \geq 0}$ to be defined as a 
zero-delayed random walk with increment distribution $\mu_{\alpha}:=\mathrm{E} \sum_{|v|=1} \mathrm{e}^{-\alpha S(v)} \delta_{S(v)}$. Our main assumptions (A1) and (A2) ensure that $\mu_{\alpha}$ is a probability measure and that

$$
\mu:=\mathrm{E} S_{1}=\int x \mu_{\alpha}(\mathrm{d} x)=\mathrm{E} \sum_{i=1}^{N} S(i) \mathrm{e}^{-\alpha S(i)}=-m^{\prime}(\alpha) \in(0, \infty),
$$

i.e. that the associated random walk has finite positive drift. Here, for our convenience, we implicitly assume that the random walk $\left(S_{n}\right)_{n \geq 0}$ is defined on the probability space $(\Omega, \mathcal{A}, \mathrm{P})$. The announced connection between the BRW and its associated random walk is established as follows. For $v \in g$, we define $S(v):=(S(\varnothing), S(v \mid 1), \ldots, S(v))$. Then, for any Borel set $B \subseteq \mathbb{R}^{n+1}$,

$$
\mathrm{P}\left(\left(S_{0}, \ldots, S_{n}\right) \in B\right)=\mathrm{E} \sum_{|v|=n} \mathrm{e}^{-\alpha S(v)} \delta_{S(v)}(B) .
$$

A proof of this formula can be found in [4, Lemma 4.1].

Remark 5.1. Condition 3.1 and the second part of Condition 3.2 can be interpreted as moment assumptions on the associated random walk $\left(S_{n}\right)_{n \geq 0}$. Indeed, via (5.1), Condition 3.1 is equivalent to $\mathrm{E} 1 / \mathrm{g}\left(S_{1}^{+}\right)<\infty$, whereas the second part of Condition 3.2 is equivalent to $S_{1}^{-}$ having a finite $x^{2}(\log x)^{1+\varepsilon}$-moment. In applications, a typical choice of $g$ would also be $1 / g(x)=1 \vee|x|\left(\log ^{+}|x|\right)^{1+\varepsilon}, x \in \mathbb{R}$, which would make the condition on $S_{1}^{+}$equivalent to $S_{1}^{+}$having an $x(\log x)^{1+\varepsilon}$-moment.

\section{Ladder lines and ladder heights}

The main idea in the proofs of Theorems 3.1 and 3.2 is to reduce the convergence result for BRWs on the real line to BRWs with positive steps via ladder lines.

To this end, define the first strictly ascending ladder line $g_{1}^{>}$by

$$
g_{1}^{>}:=\{v \in g: S(v)>0 \text { and } S(u) \leq 0 \text { for all } u \prec v\} .
$$

Then, for any $v \in \mathbb{V}$, by the definition of the bracket notation $[\cdot]_{v}$ in Section $2,\left[\mathcal{g}_{1}^{>}\right]_{v}$ is the corresponding line for the BRW rooted in $v$. Now we define $g_{0}>:=\{\varnothing\}$, and, recursively, for $n \geq 1$,

$$
g_{n}^{>}:=\left\{v w: v \in g_{n-1}^{>}, w \in\left[g_{1}^{>}\right]_{v}\right\}
$$

Clearly, the $g_{n}^{>}$are random subsets of $\mathbb{V}$. They are special optional lines in the sense of [10], and [5], where stopping along any line of descent follows the same rule. Indeed, we have $v \in g_{n}^{>}$if and only if $S(v)$ is the $n$th strict record in the finite sequence $S(\varnothing), S(v \mid 1), \ldots, S(v)$. Therefore, we refer to $g_{n}>$ as the $n$th strictly ascending ladder line. This ladder line corresponds to the $n$th strictly ascending ladder index in classical renewal theory. To be more precise about this connection, let $\sigma^{>}: \mathbb{R}^{\mathbb{N}_{0}} \rightarrow \mathbb{N}_{0} \cup\{\infty\}$ be defined by

$$
\sigma^{>}\left(\left(s_{k}\right)_{k \geq 0}\right):=\inf \left\{k \geq 0: s_{k}>0\right\} .
$$

For $n \in \mathbb{N}_{0}$, let the formal stopping rule $\sigma_{n}^{>}$denote the $n$th consecutive application of $\sigma^{>}$, which means that $\sigma_{0}^{>}:=0$ and

$$
\sigma_{n}^{>}\left(\left(s_{k}\right)_{k \geq 0}\right):=\inf \left\{k>\sigma_{n-1}^{>}: s_{k}-s_{\sigma_{n-1}^{>}}>0\right\}, \quad n \in \mathbb{N} .
$$

In other words, $\sigma_{n}^{>}\left(\left(s_{k}\right)_{k \geq 0}\right)$ is the index of the $n$th strict record in the sequence $\left(s_{k}\right)_{k \geq 0}$. As usual, we stipulate that inf $\varnothing=\infty$. Since subpopulations of the BRW may become extinct, we 
have to extend the definition of $\sigma_{n}^{>}$to finite sequences of reals $s=\left(s_{0}, \ldots, s_{k}\right) \in \mathbb{R}^{k+1}, k \geq 0$. Here, we define $\sigma_{n}^{>}(s)$ to be $j$ if $s_{j}$ is the $n$th strict record in the tuple $s$. If no such record exists, we define $\sigma_{n}^{>}(s):=\infty$. With this definition, we have

$$
g_{n}^{>}=\left\{v \in g: \sigma_{n}^{>}\left((S(v \mid k))_{0 \leq k \leq|v|}\right)=|v|\right\} .
$$

We use these lines to construct an embedded BRW $\left(\mathcal{Z}_{n}^{>}\right)_{n \geq 0}$ with positive steps by defining

$$
\mathcal{Z}_{n}^{>}:=\sum_{v \in \mathscr{G}_{n}^{>}} \delta_{S(v)}, \quad n \geq 0
$$

Jagers [10] proved that the branching property, which is the property that, given $\mathcal{F}_{n}$, for different individuals $v$ in generation $n$, the BRWs based on the point processes $\mathcal{Z}(v w), w \in \mathbb{V}$, are independent copies of the original BRW, carries over to optional lines. In particular, this is true for the strictly ascending ladder lines. To be more precise, define $\mathcal{F}_{n}{ }^{>}$to be the $\sigma$-algebra generated by the sets of the form $\left\{p_{v} \in A\right\} \cap\left\{v \prec g_{n}^{>}\right\}, A \in \mathcal{A}_{v}$, where $p_{v}: \Omega \rightarrow \Omega_{v}$, $p_{v}\left(\left(\omega_{u}\right)_{u \in \mathbb{V}}\right)=\omega_{v}$. Then the families $(\mathcal{Z}(v w))_{w \in \mathbb{V}}, v \in \mathscr{g}_{n}^{>}$, are conditionally i.i.d. given $\mathcal{F}_{n}^{>}$ and distributed like $(\mathcal{Z}(w))_{w \in \mathbb{V}}$; see [10, Theorem 4.14]. Therefore, $\left(\mathcal{Z}_{n}^{>}\right)_{n \geq 0}$ forms a BRW, which clearly has positive steps. As in [2, Section 3], we define $\Upsilon_{n, k}^{>}:=\pi_{0: k}\left(\left\{\sigma_{n}^{>}=k\right\}\right)$, where $\pi_{0: k}: \mathbb{R}^{\mathbb{N}_{0}} \rightarrow \mathbb{R}^{k+1}$ denotes the projection $\left(s_{j}\right)_{j \geq 0} \mapsto\left(s_{0}, \ldots, s_{k}\right)$ and $\left\{\sigma_{n}^{>}=k\right\}$ is shorthand for $\left\{s \in \mathbb{R}^{\mathbb{N}_{0}}: \sigma_{n}^{>}(s)=k\right\}$. Then, by (5.1), for any Borel set $B \subseteq \mathbb{R}^{k+1}$,

$$
\begin{aligned}
\mathrm{P}\left(\left(S_{0}, \ldots, S_{k}\right) \in B, \sigma_{n}^{>}=k\right) & =\mathrm{P}\left(\left(S_{0}, \ldots, S_{k}\right) \in B \cap \Upsilon_{n, k}^{>}\right) \\
& =\mathrm{E} \sum_{|v|=k} \mathrm{e}^{-\alpha S(v)} \delta_{S(v)}\left(B \cap \Upsilon_{n, k}^{>}\right) \\
& =\mathrm{E} \sum_{\left\{|v|=k: v \in \mathcal{G}_{n}^{>}\right\}} \mathrm{e}^{-\alpha S(v)} \delta_{S(v)}(B),
\end{aligned}
$$

where in slight abuse of notation we write $\sigma_{n}^{>}$instead of $\sigma_{n}^{>}\left(\left(S_{j}\right)_{j \geq 0}\right)$ in the first line of (6.4). Choosing $B=\mathbb{R}^{k} \times A$ for an arbitrary Borel set $A \subseteq \mathbb{R}$ and summation over $k \geq 0$ now yield

$$
\mu_{\alpha, n}^{>}(A):=\mathrm{E} \sum_{v \in g_{n}^{>}} \mathrm{e}^{-\alpha S(v)} \delta_{S(v)}(A)=\mathrm{P}\left(S_{\sigma_{n}^{>}} \in A, \sigma_{n}^{>}<\infty\right) .
$$

Under assumption (A2), $0<-m^{\prime}(\alpha)=\mathrm{E} S_{1}$ so that $\sigma_{n}^{>}<\infty$ a.s. for all $n$ and (6.5) simplifies to

$$
\mu_{\alpha, n}^{>}(\cdot)=\mathrm{E} \sum_{v \in g_{n}>} \mathrm{e}^{-\alpha S(v)} \delta_{S(v)}(\cdot)=\mathrm{P}\left(S_{\sigma_{n}^{>}} \in \cdot\right) .
$$

We have thus identified the distribution of the associated random walk of the embedded BRW with generations $g_{n}^{>}, n \geq 0$. As a particular consequence of (6.6), we obtain $m^{>}(\alpha)=$ $\mu_{\alpha, n}^{>}(\mathbb{R})=\mathrm{P}\left(S_{\sigma_{n}}>\in \mathbb{R}\right)=1$, where $m^{>}$denotes the Laplace transform of the intensity measure of $\mathfrak{Z}_{1}^{>}$. In other words, assumption (A1) carries over from the original BRW $\left(Z_{n}\right)_{n \geq 0}$ to the embedded BRW $\left(\mathcal{Z}_{n}^{>}\right)_{n \geq 0}$. Furthermore, an application of Wald's equations yields

$$
\mu^{>}:=\mathrm{E} S_{\sigma^{>}}=\mu \mathrm{E} \sigma^{>} \in(0, \infty),
$$

i.e. (A2) carries over too. From [3, Lemma 8.1] (with $T_{i}:=\mathrm{e}^{-S(i)}$ if $i \leq N$ and $T_{i}:=0$ otherwise), we infer that $\mathrm{E} N^{>}>1$ for $N^{>}:=\mathcal{Z}^{>}(\mathbb{R})$, and furthermore that $\left(\mathcal{Z}_{n}^{>}\right)_{n \geq 0}$ is 
nonlattice if and only if the same is true for $\left(\mathcal{Z}_{n}\right)_{n \geq 0}$. Finally, in the case that $\left(\mathcal{Z}_{n}\right)_{n \geq 0}$ is lattice, then so is $\left(\mathcal{Z}_{n}^{>}\right)_{n \geq 0}$ and even the lattice spans coincide in this case.

Next, we check that Condition 3.1 carries over from the original BRW to the corresponding ladder line process.

Lemma 6.1. If $\left(\mathcal{Z}_{n}\right)_{n \geq 0}$ satisfies Condition 3.1 then so does $\left(\mathcal{Z}_{n}^{>}\right)_{n \geq 0}$.

Proof. The following estimation makes use of (6.6), the fact that $1 / g$ is nondecreasing and positive, and the definition of $S_{1}$ :

$$
\begin{aligned}
\mathrm{E} \sum_{v \in g_{1}} \frac{\mathrm{e}^{-\alpha S(v)}}{g(S(v))} & =\mathrm{E} \frac{1}{g\left(S_{\sigma^{>}}\right)} \\
& \leq \mathrm{E} \sum_{k=1}^{\sigma^{>}} \frac{1}{g\left(\left(S_{k}-S_{k-1}\right)^{+}\right)} \\
& =\mathrm{E} \sigma^{>} \mathrm{E} \frac{1}{g\left(S_{1}^{+}\right)} \\
& =\mathrm{E} \sigma^{>} \mathrm{E} \sum_{|v|=1} \frac{\mathrm{e}^{-\alpha S(v)}}{g\left(S(v)^{+}\right)} \\
& <\infty,
\end{aligned}
$$

where the penultimate equality follows from Wald's equation and the finiteness of the last expectation is due to the validity of Condition 3.1 for $\mathcal{Z}$.

The next result in this section is a simple consequence of (6.4), which we will need in the proof of our main results in Section 7. In order to formulate the next result, we introduce the pre-g, $\mathcal{Q}_{1}^{>}$occupation measure $\mathcal{V}^{>}$of $\left(\mathcal{Z}_{n}\right)_{n \geq 0}$ :

$$
\mathcal{V}^{>}:=\sum_{v \prec g_{1}} \mathrm{e}^{-\alpha S(v)} \delta_{S(v)},
$$

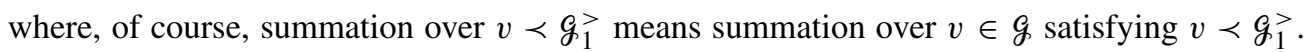
Needless to say that $\mathcal{V}^{>}$is connected with the pre- $\sigma^{>}$occupation measure $V^{>}$of the associated random walk $\left(S_{n}\right)_{n \geq 0}$.

Lemma 6.2. For any Borel set $B \subseteq \mathbb{R}$, we have

$$
\mathrm{E} \mathcal{V}^{>}(B)=V^{>}(B) \text {. }
$$

Proof. Let $B \subseteq \mathbb{R}$ be a Borel subset of $\mathbb{R}$. For $n \geq 0$, we define $\Upsilon_{n}^{>}:=\pi_{0: n}\left(\left\{\sigma^{>}>n\right\}\right)$. Then, by (5.1),

$$
\begin{aligned}
\mathrm{E}^{>} \mathcal{V}^{>}(B) & =\mathrm{E} \sum_{v \prec g_{1}} \mathrm{e}^{-\alpha S(v)} \delta_{S(v)}(B) \\
& =\sum_{n \geq 0} \mathrm{E} \sum_{\left\{|v|=n: v \prec g_{1}^{>}\right\}} \mathrm{e}^{-\alpha S(v)} \delta_{S(v)}\left(\mathbb{R}^{n} \times B\right) \\
& =\sum_{n \geq 0} \mathrm{E} \sum_{|v|=n} \mathrm{e}^{-\alpha S(v)} \delta_{S(v)}\left(\left(\mathbb{R}^{n} \times B\right) \cap \Upsilon_{n}^{>}\right) \\
& =\sum_{n \geq 0} \mathrm{P}\left(\left(S_{0}, \ldots, S_{n}\right) \in\left(\mathbb{R}^{n} \times B\right) \cap \Upsilon_{n}^{>}\right)
\end{aligned}
$$




$$
\begin{aligned}
& =\sum_{n \geq 0} \mathrm{P}\left(S_{n} \in B, \sigma^{>}>n\right) \\
& =\mathrm{E} \sum_{n=0}^{\sigma^{>}-1} \mathbf{1}_{B}\left(S_{n}\right) \\
& =V^{>}(B) .
\end{aligned}
$$

Remark 6.1. It is worth mentioning that though $g_{1}^{>}$may in general consist of infinitely many individuals with positive probability, the number of ancestors of $g_{1}^{>}, \#\left\{v \prec g_{1}^{>}\right\}$, is integrable and hence finite with probability 1 . To see this, we estimate as follows:

$$
\mathrm{E} \#\left\{v \prec g_{1}^{>}\right\} \leq \mathrm{E} \sum_{v \prec g_{1}^{>}} \mathrm{e}^{-\alpha S(v)}=\mathrm{E} \mathcal{V}^{>}(\mathbb{R})=V^{>}(\mathbb{R})=\mathrm{E} \sigma^{>}<\infty .
$$

\section{Proof of the main result (Theorems 3.1 and 3.2)}

Without loss of generality, we assume that $\phi(t, \omega) \geq 0$ for all $t \in \mathbb{R}, \omega \in \Omega$. Otherwise, we can derive Theorems 3.1 and 3.2 for $\phi^{+}$and $\phi^{-}$instead of $\phi$, and put the results together afterwards. The proof of our main result (Theorems 3.1 and 3.2) is divided into two steps. The starting point of our proof is the observation that Theorems 3.1 and 3.2 hold true under the additional assumptions that $\mathbb{Z}$ is concentrated on $[0, \infty)$ and that $\phi$ vanishes on the negative half-line; see Remarks 3.2 and 3.3. In our first step, we remove the assumption that $\phi$ vanishes on the negative half-line. In the second step, the almost-sure renewal theorems will be extended to general $\mathcal{Z}$. In both steps, we deal with the nonlattice case only. The lattice case is similar; the corresponding details are left to the reader.

Step 1. In this step we show that if (3.5) and (3.6) respectively hold for characteristics $\phi$ vanishing on the negative half-line (but fulfilling Condition 3.2), then the convergence also holds for any characteristic $\phi$ satisfying Condition 3.2. To this end, let $\phi$ denote a process satisfying Condition 3.2, but not necessarily vanishing on the negative half-line. Then fix any $n \in \mathbb{N}_{0}$, and define

$$
\phi_{n, 1}(t):=\mathbf{1}_{[-n, \infty)}(t) \phi(t), \quad t \in \mathbb{R},
$$

and

$$
\phi_{n, 2}(t):=\mathbf{1}_{(-\infty,-n)}(t) \phi(t), \quad t \in \mathbb{R},
$$

so that

$$
Z_{t}^{\phi}=Z_{t}^{\phi_{n, 1}}+Z_{t}^{\phi_{n, 2}}, \quad t \in \mathbb{R} .
$$

From Theorem 5.4 of [13] we know that, as $t$ tends to $\infty, Z_{t}^{\phi_{n, 1}}$ exhibits the behaviour described by (3.5), i.e.

$$
\begin{aligned}
\mathrm{e}^{-\alpha t} Z_{t}^{\phi_{n, 1}} & =\mathrm{e}^{-\alpha t} \sum_{v}[\phi]_{v}(t-S(v)) \mathbf{1}_{[-n, \infty)}(t-S(v)) \\
& =\mathrm{e}^{\alpha n} \mathrm{e}^{-\alpha(t+n)} \sum_{v}[\phi]_{v}(t+n-S(v)-n) \mathbf{1}_{[0, \infty)}(t+n-S(v)) \\
& \rightarrow \mathrm{e}^{\alpha n} \frac{W^{(\alpha)}}{\mu} \int_{0}^{\infty} \mathrm{e}^{-\alpha s} \mathrm{E} \phi(s-n) \mathrm{d} s \\
& =\frac{W^{(\alpha)}}{\mu} \int_{-n}^{\infty} \mathrm{e}^{-\alpha s} \mathrm{E} \phi(s) \mathrm{d} s \quad \text { a.s. as } t \rightarrow \infty .
\end{aligned}
$$


To estimate the second summand on the right-hand side of (7.3), we introduce one further characteristic $\psi$, defined by

$$
\psi(t):=M \mathbf{1}_{[0,1)}(t), \quad t \in \mathbb{R} .
$$

(Recall that $M$ is defined in Condition 3.2.) Then, again by (3.5), we obtain

$$
\mathrm{e}^{-\alpha t} Z_{t}^{\psi} \rightarrow \frac{W^{(\alpha)}}{-m^{\prime}(\alpha)} \int_{0}^{1} \mathrm{e}^{-\alpha s} \mathrm{E} M \mathrm{~d} s \quad \text { a.s. as } t \rightarrow \infty .
$$

In particular, with probability 1 , we can choose $n_{0} \in \mathbb{N}$ such that

$$
\mathrm{e}^{-\alpha t} Z_{t}^{\psi} \leq \frac{(\mathrm{E} M) W^{(\alpha)}}{-m^{\prime}(\alpha)}+1 \text { for all } t \geq n_{0} .
$$

Thus,

$$
\begin{aligned}
\mathrm{e}^{-\alpha t} Z_{t}^{\phi_{n, 2}} & =\mathrm{e}^{-\alpha t} \sum_{v}[\phi]_{v}(t-S(v)) \mathbf{1}_{(-\infty,-n)}(t-S(v)) \\
& =\sum_{v} \mathrm{e}^{-\alpha S(v)} \frac{\mathrm{e}^{-\alpha(t-S(v))}[\phi]_{v}(t-S(v))}{h(t-S(v))} h(t-S(v)) \mathbf{1}_{(-\infty,-n)}(t-S(v)) \\
& \leq \sum_{k>n} \sum_{v} \mathrm{e}^{-\alpha S(v)}[M]_{v} h(-(k-1)) \mathbf{1}_{[-k,-(k-1))}(t-S(v)) \\
& \leq \mathrm{e}^{\alpha} \sum_{k>n} h(-(k-1)) \mathrm{e}^{-\alpha(t+k)} \sum_{v}[M]_{v} \mathbf{1}_{[0,1)}(t+k-S(v)) \\
& =\mathrm{e}^{\alpha} \sum_{k>n} h(-(k-1)) \mathrm{e}^{-\alpha(t+k)} Z_{t+k}^{\psi} \\
& \leq \mathrm{e}^{\alpha}\left(\frac{(\mathrm{E} M) W^{(\alpha)}}{-m^{\prime}(\alpha)}+1\right) \sum_{k \geq n} h(-k)
\end{aligned}
$$

for all sufficiently large $n$ and all $t \geq 0$. In conclusion,

$$
\limsup _{t \rightarrow \infty} \mathrm{e}^{-\alpha t} Z_{t}^{\phi_{n, 2}} \leq \mathrm{e}^{\alpha} \frac{(\mathrm{E} M) W^{(\alpha)}}{-m^{\prime}(\alpha)+1} \sum_{k \geq n} h(-k) \quad \text { a.s. }
$$

The latter term tends to 0 as $n \rightarrow \infty$ due to the integrability of $h$. In view of (7.3) and (7.4), we arrive at

$$
\lim _{t \rightarrow \infty} \mathrm{e}^{-\alpha t} Z_{t}^{\phi}=\frac{W^{(\alpha)}}{-m^{\prime}(\alpha)} \int_{-\infty}^{\infty} \mathrm{e}^{-\alpha s} \operatorname{E} \phi(s) \mathrm{d} s \quad \text { a.s. }
$$

Step 2. In the final step, we remove the assumption that $\mathbb{Z}$ is concentrated on the positive half-line. Let $\phi$ denote a process satisfying Condition 3.2. In what follows, we make use of the ladder line concept introduced in Section 6. Recall that $\left(\mathcal{Z}_{n}^{>}\right)_{n \geq 0}$ denotes the embedded BRW of strictly ascending ladder heights. For any characteristic $\psi$, we define

$$
Z^{>, \psi}(t):=\sum_{v \in \mathcal{g}>}[\psi]_{v}(t-S(v)), \quad t \in \mathbb{R},
$$


that is, $Z^{>, \psi}$ denotes the general branching process counted with characteristic $\psi$ based on $\left(\mathcal{Z}_{n}^{>}\right)_{n \geq 0}$. Now we define a new characteristic $\phi^{>}$by

$$
\phi^{>}(t):=\sum_{v \prec g_{1}^{>}}[\phi]_{v}(t-S(v)), \quad t \in \mathbb{R} .
$$

Since $\left\{v w: v \in g^{>}, w \prec\left[g_{1}^{>}\right]_{v}\right\}=g, Z_{t}^{\phi}$ satisfies

$$
\begin{aligned}
Z_{t}^{\phi} & =\sum_{v \in G}[\phi]_{v}(t-S(v)) \\
& =\sum_{v \in G>} \sum_{w<\left[g_{1}^{>}\right]_{v}}[\phi]_{v w}(t-S(v w)) \\
& =\sum_{v \in G^{>}}\left[\phi^{>}\right]_{v}(t-S(v)) \\
& =Z_{t}^{>>\phi^{>}}, \quad t \in \mathbb{R} .
\end{aligned}
$$

Thus, $Z^{\phi}$ can be interpreted as a general branching process counted with characteristic $\phi^{>}$in the sense of Nerman [13]. We intend to apply the known results of Nerman and Gatzouras to derive the limiting behaviour of $Z_{t}^{\phi}=Z_{t}^{>, \phi^{>}}$.

To this end, recall that we restrict ourselves to the nonlattice case and note that in this case $\phi$ is assumed to have càdlàg paths with probability 1 . Then so has $[\phi]_{v}$ for any $v \in \mathbb{V}$. Furthermore, by Remark 6.1, $\left\{v \prec g_{1}^{>}\right\}$is finite with probability 1 . Thus, $\phi^{>}(\cdot)=\sum_{v \prec g_{1}^{>}}[\phi]_{v}(\cdot-S(v))$ also has càdlàg paths.

Moreover, for any $t \geq 0$, using $S(v) \leq 0$ on $\left\{v \prec g_{1}^{>}\right\}$and the monotonicity of $h$ on $[0, \infty)$, we obtain

$$
\begin{aligned}
\frac{\mathrm{e}^{-\alpha t} \phi^{>}(t)}{h(t)} & =\sum_{v \prec g_{1}^{>}} \mathrm{e}^{-\alpha S(v)} \frac{\mathrm{e}^{-\alpha(t-S(v))}[\phi]_{v}(t-S(v))}{h(t-S(v))} \frac{h(t-S(v))}{h(t)} \\
& \leq \sum_{v<g_{1}^{>}} \mathrm{e}^{-\alpha S(v)}[M]_{v},
\end{aligned}
$$

where $M$ is defined in Condition 3.2. Thus,

$$
\mathrm{E} \sup _{t \geq 0} \frac{\mathrm{e}^{-\alpha t} \phi^{>}(t)}{h(t)} \leq \mathrm{E} \sum_{v<g_{1}^{>}} \mathrm{e}^{-\alpha S(v)}[M]_{v}=\mathrm{E} \sigma^{>} \mathrm{E} M<\infty .
$$

For $t \leq 0$, the fraction $h(t-S(v)) / h(t)$ appearing in (7.6) is no longer bounded by 1 for all possible values of $S(v) \leq 0$. In fact, if $S(v)$ is close to $t$ then the fraction $h(t-S(v)) / h(t)$ is of the order $h(0) / h(S(v))$, which is the worst that can happen. But since $h$ is regularly varying at $-\infty$ with index -1 , we have $h(t / 2) / h(t) \rightarrow 2$ as $t \rightarrow \infty$. Thus,

$$
C:=\sup _{t \leq 0} \frac{h(t / 2)}{h(t)}<\infty .
$$

Since $h$ is increasing on $(-\infty, 0]$, we have

$$
\frac{h(t-S(v))}{h(t)} \leq \frac{h(t / 2)}{h(t)} \leq C \quad \text { on }\left\{S(v) \geq \frac{t}{2}\right\}
$$


Consequently,

$$
\begin{aligned}
\frac{\mathrm{e}^{-\alpha t} \phi^{>}(t)}{h(t)} & \leq \sum_{v \prec g_{1}} \mathrm{e}^{-\alpha S(v)}[M]_{v} \frac{h(t-S(v))}{h(t)} \\
& \leq \sum_{v \prec g_{1}^{>}} \mathrm{e}^{-\alpha S(v)}[M]_{v}\left(C+\frac{h(t-S(v))}{h(t)} \mathbf{1}_{\{S(v) \leq t / 2\}}\right) \\
& \leq \sum_{v<g_{1}} \mathrm{e}^{-\alpha S(v)}[M]_{v}\left(C+\frac{h(0)}{h(2 S(v))}\right) .
\end{aligned}
$$

Due to the fact that $h$ is regularly varying at $-\infty$, the series in inequality (7.7) is integrable with respect to $\mathrm{P}$ if the same is true for $\sum_{v<g>} \mathrm{e}^{-\alpha S(v)}[M]_{v} / h(S(v))$. For $v \in \mathbb{V},|v|=n$, we define $\tilde{F}_{\prec v}$ to be the $\sigma$-algebra generated by all projections $p_{u}, u \prec v$. Then $\mathbf{1}_{\left\{v \in g, v<g_{1}\right\}} f(S(v))$ is $\mathcal{F}_{v}$-measurable for any measurable function $f \geq 0$, whereas $(\mathcal{Z}(v w))_{w \in \mathbb{V}}$ is independent of $\mathcal{F}_{\prec v}$. Hence,

$$
\begin{aligned}
\mathrm{E} \sum_{v \prec g_{1}} \mathrm{e}^{-\alpha S(v)}[M]_{v} \frac{1}{h(S(v))} & =\sum_{v \in \mathbb{V}} \mathrm{E}\left(\mathrm{E}\left[\mathbf{1}_{\left\{v \in g, v<g_{1}^{>}\right\}} \mathrm{e}^{-\alpha S(v)}[M]_{v} \frac{1}{h(S(v))} \mid \mathcal{F}_{\prec v}\right]\right) \\
& =\sum_{v \in \mathbb{V}} \mathrm{E}\left(\mathbf{1}_{\left\{v \in g, v<g_{1}^{>}\right\}} \frac{\mathrm{e}^{-\alpha S(v)}}{h(S(v))}(\mathrm{E} M)\right) \\
& =(\mathrm{E} M) \mathrm{E} \sum_{v<g_{1}^{>}} \frac{\mathrm{e}^{-\alpha S(v)}}{h(S(v))} \\
& =(\mathrm{E} M) \int \frac{1}{h(s)} V^{>}(\mathrm{d} s),
\end{aligned}
$$

where the last equality follows from Lemma 6.2. The latter integral is finite due to Condition 3.2 and Proposition 4.1. Gathering the facts above, we have shown that $\mathcal{Z}^{>}$and $\phi^{>}$fulfill the assumptions of Nerman's almost-sure renewal theorem in the version we have established in step 1. Hence, with $\bar{\phi}(t):=\mathrm{E} \phi(t)$, we obtain

$$
\begin{aligned}
\mathrm{e}^{-\alpha t} Z_{t}^{\phi} & \rightarrow \frac{W^{(\alpha)}}{\mu^{>}} \int_{-\infty}^{\infty} \mathrm{e}^{-\alpha s} \mathrm{E} \phi^{>}(s) \mathrm{d} s \\
& =\frac{W^{(\alpha)}}{\mu^{>}} \int_{-\infty}^{\infty} \mathrm{E} \sum_{v<g_{1}} \mathrm{e}^{-\alpha S(v)} \mathrm{e}^{-\alpha(s-S(v))}[\phi]_{v}(s-S(v)) \mathrm{d} s \\
& =\frac{W^{(\alpha)}}{\mu^{>}} \int_{-\infty}^{\infty} \mathrm{E} \sum_{k=0}^{\sigma^{>}-1} \mathrm{e}^{-\alpha\left(s-S_{k}\right)} \bar{\phi}\left(s-S_{k}\right) \mathrm{d} s \\
& =\frac{W^{(\alpha)}}{\mu^{>}} \mathrm{E} \sum_{k=0}^{\sigma^{>}-1} \int_{-\infty}^{\infty} \mathrm{e}^{-\alpha s} \bar{\phi}(s) \mathrm{d} s \\
& =\frac{W^{(\alpha)}}{\mu} \int_{-\infty}^{\infty} \mathrm{e}^{-\alpha s} \bar{\phi}(s) \mathrm{d} s \quad \text { a.s. as } t \rightarrow \infty,
\end{aligned}
$$

where in the last step we utilized (6.7). Taking into account the fact that $\mu=-m^{\prime}(\alpha)$, the proof is herewith complete. 


\section{Acknowledgements}

I am grateful to Alexander Iksanov for suggesting that I write this paper. I also wish to thank an anonymous referee for a very careful reading of the manuscript.

\section{References}

[1] Alsmeyer, G. (1991). Erneuerungstheorie. Teubner, Stuttgart (in German).

[2] Alsmeyer, G. And Meiners, M. (2008). A note on the transience of critical branching random walks on the line. In Proc. 5th Colloquium on Mathematics and Computer Science, Assoc. Discrete Math. Theoret. Comput. Sci. Nancy, pp. 421-435.

[3] Alsmeyer, G., Biggins, J. D. And Meiners, M. (2010). The functional equation of the smoothing transform. Preprint. Available at http://arxiv.org/abs/0906.3133v2.

[4] Biggins, J. D. And Kyprianou, A. E. (1997). Seneta-Heyde norming in the branching random walk. Ann. Prob. 25, 337-360.

[5] Biggins, J. D. And Kyprianou, A. E. (2004). Measure change in multitype branching. Adv. Appl. Prob. 36, 544-581.

[6] Bingham, N. H., Goldie, C. M. And Teugels, J. L. (1989). Regular Variation (Encyclopedia Math. Appl. 27). Cambridge University Press.

[7] Gatzouras, D. (2000). Lacunarity of self-similar and stochastically self-similar sets. Trans. Amer. Math. Soc. 352, 1953-1983.

[8] Gatzouras, D. (2000). On the lattice case of an almost-sure renewal theorem for branching random walks. Adv. Appl. Prob. 32, 720-737.

[9] Jagers, P. (1975). Branching Processes with Biological Applications. Wiley-Interscience, London.

[10] Jagers, P. (1989). General branching processes as Markov fields. Stoch. Process. Appl. 32, 183-212.

[11] JANSON, S. (1986). Moments for first-passage and last-exit times, the minimum, and related quantities for random walks with positive drift. Adv. Appl. Prob. 18, 865-879.

[12] LyONs, R. (1997). A simple path to Biggins' martingale convergence for branching random walk. In Classical and Modern Branching Processes (Minneapolis, MN, 1994; IMA Vol. Math. Appl. 84), Springer, New York, pp. 217-221.

[13] Nerman, O. (1981) On the convergence of supercritical general (C-M-J) branching processes. Z. Wahrscheinlichkeitsth. 57, 365-395. 